\title{
Sustitución total de la harina de pescado por subproductos avícolas suplementados con aminoácidos en dietas para juveniles de Piaractus brachypomus, Cuvier 1818
}

\author{
The total substitution of fishmeal for poultry by-product meal \\ supplemented with amino acids in diets for young Piaractus \\ brachypomus, Cuvier 1818
}

\section{Substituição total da farinha de peixe por subprodutos avícolas suplementados com aminoácidos em dietas para juvenis de Piaractus brachypomus, Cuvier 1818}

\section{AlvaroJ. Piñeros-Roldan ' Mariana C. Gutiérrez-Espinosa ${ }^{2}$; Segundo R. Castro-Guerrero ${ }^{3}$}

MVZ, Esp, Instituto de Acuicultura de los Llanos, Facultad de Ciencias Agropecuarias y Recursos Naturales, Universidad de los Llanos, Villavicencio, Colombia.

Zootecnista, Esp, MSc, Docente Instituto de Acuicultura de los Llanos, Facultad de Ciencias Agropecuarias y Recursos Naturales, Universidad de los Llanos, Villavicencio, Colombia.

MVZ, Esp. Escuela de Medicina Veterinaria y Zootecnia, Facultad de Ciencias Agropecuarias, Universidad Pedagógica y Tecnológica de Colombia, Tunja, Colombia.

Email: javiermvz@outlook.com

\section{Resumen}

El objetivo del presente estudio fue determinar si la harina de subproductos avícolas puede sustituir la harina de pescado como ingrediente proteico en los alimentos balanceados para juveniles de Piaractus brachypomus en condiciones controladas. Fueron formuladas tres dietas isoproteícas e isoenergéticas con 34\% de PB y $4160 \mathrm{Kcal} / \mathrm{kg}$ EB; el tratamiento 1 (T1) contenía como ingrediente proteico 30\% de harina de pescado; el tratamiento 2 (T2), 30\% de harina de subproductos avícolas; y el tratamiento 3 (T3), 30\% de harina de subproductos avícolas suplementada con aminoácidos (Lisina, Metionina y Treonina). Se seleccionaron 240 alevinos de peso promedio de $3 \mathrm{gr}$ y se distribuyeron aleatoriamente en 12 tanques de 500 litros (3 tratamientos por 4 réplicas). Se midieron parámetros productivos (ganancia de peso, consumo de alimento, tasa de conversión alimenticia, tasa específica de crecimiento) y costos de las dietas empleadas. La ganancia de peso $(1233,2 \pm 156,1 \%)$, tasa específica de crecimiento $(5,06 \pm 2 \%)$ y la tasa de conversión alimenticia $(1,22 \pm 0,09 \mathrm{gr})$ no presentaron diferencias significativas entre tratamientos. El consumo de alimento fue significativamente menor en la dieta 1, respecto a las otras. En cuanto a los costos, T1 tuvo menor valor que los otros tratamientos, los cuales se vieron aumentados en 4 y 5,8\%, para T3 y T2 respectivamente, con lo cual se muestra que los costos son similares y dependen de la situación en el mercado de los diferentes ingredientes de los que se componen las dietas. Los resultados del presente estudio indican que la harina de subproductos avícolas puede ser usada para la alimentación de 
juveniles de cachama Blanca sin repercusiones en la ganancia de peso, en la tasa de conversión alimenticia y en la tasa especifica de crecimiento, y ya que la suplementación de aminoácidos no brinda efectos significativos, no hace necesario su uso en estas dietas para la especie.

Palabras claves: Aminoácidos, Fuentes Alternativas, Nutrición, Peces tropicales, Suplementación, cachama Blanca.

\begin{abstract}
The aim of the present study was to determinate if poultry by-product meal (PBM) could substitute fish meal as protein ingredient in balanced diets for young Piaractus brachypomus (known as red-bellied pacu or white cachama) in controlled conditions. Three isoprotein/isoenergetic diets were formulated with 34\% PBM and 4,160 Kcal/kg EB; treatment 1 (T1) contained 30\% fishmeal as protein ingredient, treatment 2 (T2) 30\% PBM and treatment 3 (T3) 30\% PBM supplemented with aminoacids (lysine, methionine and threonine). 240 fry were selected, weighing $3 \mathrm{gr}$ on average, and randomly distributed in 12 tanks each one with 500 litres (3 treatments per 4 repeats). Production parameters were measured (weight gain, feed intake, feed conversion rate, specific growth rate) and the cost of the diets being used. There were no significant differences between treatments regarding weight gain $(1,233.2 \pm 156.1 \%)$, specific growth rate $(5.06 \pm 2 \%)$ and feed conversion rate $(1.22 \pm 0.09 \mathrm{gr})$. Feed in take was significantly lower in diet 1, compared to the other diets. Concerning costs, T1 was cheaper than the other treatments which increased by $4 \%$ for T 3 and 5,8\% for T2, there by showing that costs were similar and depended on the market situation of the different ingredients in the diets. The present study's results indicated that PBM could be used for feeding young white cachama without producing a negative effect concerning weight gain, feed conversion rate and specific growth rate and, since aminoacid supplements had no significant effects, using them on these diets for the species should not be necessary.
\end{abstract}

Key words: aminoacid, alternative source, nutrition, tropical fish, dietary supplement, white cachama/red-bellied pacu.

\title{
Resumo
}

O objetivo deste estudo foi determinar a possibilidade de substituir a farinha de peixe como ingrediente proteico nos alimentos balanceados para juvenis de Piaractus brachypomus em condições controladas. Foram formuladas três dietas isoprotéicas com $34 \%$ de PB e isoenergéticas com $4160 \mathrm{kcal} / \mathrm{kg} \mathrm{EB}$, o tratamento 1 (T1) continha como ingrediente proteico $30 \%$ de farinha de peixe; o tratamento 2 (T2), 30\% de farinha de subprodutos avícolas; e o tratamento 3 (T3), 30\% de farinha de subprodutos avícolas suplementado com aminoácidos (lisina, metionina e treonina). Foram selecionados 240 alevines de peso médio de $3 g$ e distribuídos ao acaso em 12 tanques de 500 litros (3 tratamentos por 4 réplicas). Os parâmetros produtivos avaliados foram (ganho de peso, consumo de ração, taxa de conversão, taxa especifica de crescimento) e custos das dietas usadas. O ganho de peso $(1233,2 \pm 156,1 \%)$ taxa especifica de crescimento $(5,06 \pm 2 \%)$ e taxa de conversão de alimentícia $(1,22 \pm 0,09$ g) não presentaram diferenças significativas entre tratamentos. O consumo de alimento foi menor significativamente na dieta 1, comparada com ás outras. Em quanto aos custos, t1 teve menor valos que os outros tratamentos, os quais aumentaram em 4 e 5,8\%, para T3 e T2 
respectivamente, demostrando que os custos são similares e dependem da situação no mercado dos ingredientes que compõem as dietas. Os resultados do presente estudo indicam que a farinha de subprodutos avícolas pode ser utilizada na alimentação de juvenis de pirapitingas em influencia no ganho de peso, na taxa de conversão alimentar e na taxa especifica de crescimento, e já que a suplementação de aminoácidos não oferece efeitos significativos, não e necessário o seu uso nas dietas para a espécie.

Palavras-chave: Aminoácidos, alternativa, nutrição, peixes tropicais, suplementação, Pirapitinga.

\section{Introducción}

La producción de dietas nutricionalmente balanceadas es el principal factor que afecta la acuacultura intensiva (Turker, et al., 2005), excediendo el $70 \%$ de los gastos totales de producción (Abimorad y Carneiro, 2004). Actualmente, las raciones empleadas en la acuicultura dependen, para su suministro proteico, de las pesqueras y la agricultura, debido a que basan su producción en la harina de pescado y en segundo lugar en la harina de soya (Martínez, et al., 1996). El costo del alimento, en años recientes, ha renovado el interés en la utilización de fuentes alternativas de proteínas para el caso de los cultivos de organismos acuáticos (Robinson 1990).

Un punto crucial en la alimentación de los organismos acuáticos es la utilización de harina de pescado en las dietas formuladas para estos (Mendoza, et al., 2000; Amaya, et al., 2007). Por lo anterior, se ha venido trabajando en substitutos de alta calidad, aunque se hace difícil encontrar un reemplazo adecuado, tanto en requerimientos nutricionales como en valor comercial (Albert et al., 2009). Sin embargo, aun cuando sea de forma parcial, existe la posibilidad de poder disminuir los niveles de harina de pescado (De la Higuera, 1985).

En la búsqueda de reemplazar la harina de pescado se ha incursionado en el uso de los subproductos avícolas, como harinas de plumas, vísceras, entre otras. Sin embargo, al encontrar deficiencias en los aminoácidos presentes en estas dietas, se sugiere que las harinas de subproductos de aves pueden sustituir completamente la harina de pescado, siempre y cuando se les adicionen los aminoácidos correspondientes (Steffens, 1994). Teniendo en cuenta que esta suplementación es costosa, se hace necesario evaluar su repercusión en los parámetros productivos de especies nativas promisorios, como lo es la cachama blanca.

Piaractus brachypomus, (Cuvier,1818), conocida comúnmente como cachama blanca, es considerada como una especie con alto potencial productivo y comercial en los sistemas de producción piscícola en aguas cálidas continentales (Mesa et al., 2007) y es nativa de las cuencas de los ríos Orinoco y Amazonas (Orozco 1990, Díaz, et al., 1995). En la actualidad no existe una dieta específica para este pez y se emplean raciones para otras especies como tilapia y trucha. Sin embargo, se reportan algunas investigaciones relacionadas con requerimientos y digestibilidad para esta especie (Torres-Agudelo y Uribe-Hosie, 1995; Vásquez-Torres, et al.,2002; Fernandes, et al.,2004; y Gutiérrez-Espinosa y Vásquez-Torres,2008; Vásquez-Torres et al.,2011; Vásquez-Torres y Aria-Castellanos, 2012)

El objeto de este trabajo fue evaluar el efecto de la sustitución de la harina de pescado por harina de subproductos avícolas, y observar su repercusión en los parámetros productivos durante la etapa de crecimiento (juveniles) en Piaractus brachypomus; del mismo modo, se

Sustitución total de la harina de pescado por subproductos avícolas suplementados con aminoácidos en dietas para juveniles de Piaractus brachypomus, Cuvier 1818 
buscó corroborar si la deficiencia de aminoácidos presente en estos subproductos afecta de alguna manera estos parámetros (ganancia de peso, consumo de alimento, tasa de conversión alimenticia, tasa especifica de crecimiento), viendo la diferencia en costo y rendimiento productivo.

\section{Materiales y métodos}

\section{Localización}

El trabajo se llevó a cabo en el Laboratorio Experimental de Alimentación y Nutrición de Peces (LEANP) de la Estación Piscícola del Instituto de Acuicultura de la Universidad de los Llanos (IALL), ubicado en el kilómetro 4 vía Puerto López, en la Vereda Barcelona de la Ciudad de Villavicencio, Departamento del Meta, Colombia (Latitud $4^{\circ} 4^{\prime} 24.95^{\prime \prime} \mathrm{N}$, Longitud $73^{\circ} 34^{\prime} 56.80^{\prime \prime}$ ); 418 m.s.n.m., con una temperatura promedio anual de $25^{\circ} \mathrm{C}$, precipitación pluvial de $4050 \mathrm{~mm}$ y $75 \%$ de humedad relativa.

Material Biológico y Unidades Experimentales Se seleccionaron 240 individuos de Piaractus Brachypomus de $3 \mathrm{~g}$ aproximados de peso, que fueron distribuidos aleatoriamente en grupos de 20 peces por cada uno de los 12 tanques de 500L de capacidad (3 tratamientos, 4 réplicas). Estos tanques fueron abastecidos con un flujo de agua de 1 litro por minuto, proveniente de un sistema cerrado compuesto por cuatro biofiltros en serie, con el fin de remover partículas en suspensión y mantener constantes los parámetros físicos y químicos del agua. Para corroborar la calidad del agua, se monitoreó semanalmente con sonda multiparamétrica (YSI, profesional plus). La aireación del agua fue permanente para mantener los niveles de oxígenos próximos a saturación (8ppm).

\section{Dietas Experimentales}

Se formularon 3 dietas experimentales prácticas isoproteícas (PD) e isoenergéticas (ED), las cuales fueron comparadas entre sí (Tabla 1). Una dieta contenía harina de pescado (Tratamiento 1 o T1) y 2 dietas sustituyeron la harina de pescado por la harina de vísceras de pollo (Tratamiento 2 o T2); una de estas se suplementó con aminoácidos (Metionina, treonina y lisina) [Tratamiento 3 o T3].

Tabla 1 Composición de ingredientes (\% en base húmeda) de las dietas prácticas.

\begin{tabular}{lccc}
\hline \hline Ingrediente & $\begin{array}{c}\text { Dieta } 1 \\
\text { (T1) }\end{array}$ & $\begin{array}{c}\text { Dieta 2 } \\
\text { (T2) }\end{array}$ & $\begin{array}{c}\text { Dieta 3 } \\
\text { (T3) }\end{array}$ \\
\hline Torta de soya & 20 & 20 & 20 \\
Torta de palmiste & 7 & 7 & 7 \\
Gluten de maíz & 1,5 & 1,5 & 1,5 \\
Harina de pescado & 30 & 0 & 0 \\
Harina vísceras pollo & 0 & 30 & 30 \\
Harina de trigo & 7 & 7 & 7 \\
Maíz amarillo & 4 & 4 & 4 \\
Harina de yuca & 2 & 2 & 2 \\
Gelatina & 0 & 2,9 & 2,4 \\
Alfacelulosa & 22,5 & 20,6 & 20,6 \\
Aceite vegetal & 3,7 & 0 & 0 \\
Vitaminas & 2,5 & 2,5 & 2,5 \\
Minerales & 2,5 & 2,5 & 2,5 \\
Treonina Artificial & 0 & 0 & 0,6 \\
Metionina Artificial & 0 & 0 & 0,1 \\
Lisina Artificial & 0 & 0 & 0,1 \\
\hline \hline
\end{tabular}


Todos los ingredientes fueron pulverizados utilizando molino de pines; mezclados y humedecidos. Posteriormente, cada una de las mezclas pasó a ser extruida en micro extrusor (Exteec®, Riberão Preto -Brasil) para obtener pellets compactos y flotantes con diámetro de $3 \mathrm{~mm}$. Las dietas se secaron en horno a $60^{\circ} \mathrm{C}$ por 3 horas y se almacenaron hasta su uso.

Los peces fueron alimentados hasta aparente saciedad con las respectivas dietas experimentales, dos veces al día (0800 y 1600 horas) por 51 días. El consumo diario de alimento se registró. Una vez a la semana se efectuó la limpieza de los tanques, extrayendo los residuos por sifoneo. Al inicio y al final del experimento, previo un ayuno de 24 horas, los peces fueron pesados.

\section{Análisis de Laboratorio}

Todos los análisis de laboratorio fueron realizados por triplicado y con base en $100 \%$ de materia seca. Por lo tanto, las muestras de las dietas fueron secadas y almacenadas en bolsas de cierre hermético hasta la realización de los análisis de contenido proteico y valor energético. Para el análisis de materia seca se puso una muestra de cada una de las dietas en el horno (Memmert) a una temperatura de $105^{\circ} \mathrm{C}$ durante 24 horas. Para determinar la proteína presente se utilizó el método Kjeldalh (Metodología patrón descrita por la AOAC, 1995). La energía bruta fue determinada en la bomba calorimétrica PARR (121EA, USA).

\section{Parámetros a evaluación}

Los siguientes parámetros fueron evaluados para cada dieta:

Ganancia de peso(WG) $\%=100 \times$ (Peso final -peso inicial) / (peso inicial)
Consumo de alimento $(\mathbf{F I}) \mathbf{g} / \mathbf{d}=$ Alimento consumido (g) / Días del experimento

Tasa de conversión alimenticia $(F C R)=$ Alimento consumido (g) / Ganancia de peso

Tasa específica de crecimiento $(\mathbf{S G R})=100 \mathrm{x}$ [Ln(peso final) - Ln(peso inicial)] / tiempo (días)

\section{Costos de las dietas}

Los costos de las dietas fueron obtenidos por cotizaciones de los diferentes ingredientes en empresas comerciales que distribuyen a nivel nacional cada uno de estos. No se tiene en cuenta valor del flete, ni impuestos ligados a estos en la posterior manufactura de las dietas.

\section{Análisis estadístico}

El diseño experimental se basó en un modelo de clasificación experimental completamente aleatorizado, efecto fijo de tipo balanceado con cuatro réplicas por tratamiento, donde se utilizaron 3 tratamientos. Los datos fueron expresados como media y sometidos a análisis de varianza. Las diferencias entre tratamientos experimentales fueron determinadas por testde Tukey. Todo el proceso estadístico fue realizado a través del Programa SPSS, bajo licencia de la Universidad Pedagógica y Tecnológica de Colombia.

\section{Resultados}

El análisis de composición proximal de las dietas experimentales se presenta en la tabla 2, en donde se muestra la similitud de las dietas usadas para el proyecto. 
Tabla 2. Composición proximal de las dietas prácticas $(n=3)$

\begin{tabular}{lccc}
\hline \hline & & Dieta \\
Análisis (\%) & $\mathbf{1}$ & $\mathbf{2}$ & $\mathbf{3}$ \\
\cline { 2 - 4 } & 97,7 & 89,4 & 90,3 \\
Materia seca & 33,2 & 34,5 & 34,4 \\
Proteína Bruta & 4117 & 4162 & 4167 \\
Energía Bruta $(\mathrm{kcal} / \mathrm{kg})$ & 5,0 & 8,0 & 8,0 \\
Lípidos & 14,8 & 12,4 & 13,0 \\
Cenizas & & & \\
\hline \hline
\end{tabular}

En consumo de alimento, el tratamiento 1 fue significativamente diferente a los tratamientos 2 y $3(\mathrm{P}<0,05)$. Se evidencia que los peces alimentados con el tratamiento 3 tuvieron un mayor consumo con respecto a los otros tratamientos, presentando el menor consumo el tratamiento 1(Figura 1).

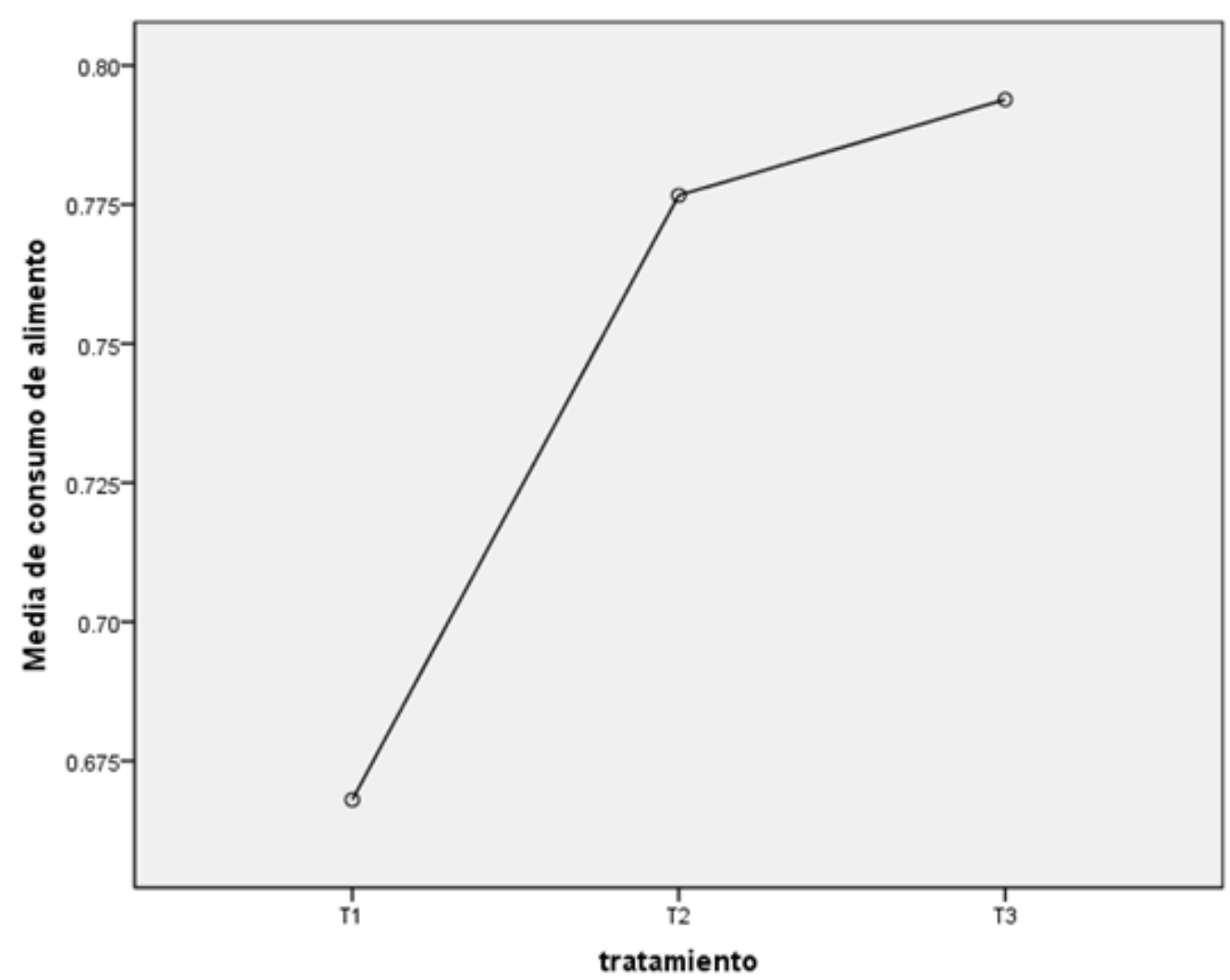

Figura 1. Consumo de Alimento Medio por pez vs Tratamiento. 
Tabla 3. Parámetros productivos de juveniles de Piaractus brachypomus, alimentados con 3 dietas experimentales

\begin{tabular}{lccc}
\hline \hline \multicolumn{1}{c}{ Parámetros } & T1 & T2 & T3 \\
\hline Ganancia de Peso (\%) & $1304,1 \pm 105,3$ & $1274,9 \pm 140,5$ & $1120,7 \pm 180,8$ \\
Consumo de Alimento Diario (g) & $0,6 \pm 0,0^{\mathrm{a}}$ & $0,7767 \pm 0714^{\mathrm{b}}$ & $0,79 \pm 0,04^{\mathrm{b}}$ \\
Tasa de Conversión alimenticia (g) & $1,1 \pm 0,08$ & $1,2 \pm 0,07$ & $1,3 \pm 0,19$ \\
Tasa Específica de Crecimiento (\%) & $5,1 \pm 0,1$ & $5,1 \pm 0,2$ & $4,8 \pm 0,3$ \\
\hline \hline
\end{tabular}

Medias seguidas por letras diferentes en las filas indican diferencias significativas entre tratamientos $(p<0.05)$.

En la tabla 4 se presentan los costos de las materias primas usadas para las dietas experimentales (estos datos están basados en cotizaciones de empresas comerciales en fechas correspondientes a la presentación del presente artículo). El menor costo se presenta en T1 (100\%), como se observa en la tabla 4, seguido de T3 y T2. En T3, se incrementa el valor en un 4\%; y para T2, este aumento es del $5,8 \%$.

Tabla 4. Costos por Kilogramo de Dietas Elaboradas para la presente investigación.

\begin{tabular}{|c|c|c|c|c|c|c|c|}
\hline \multirow{2}{*}{ Ingrediente } & \multirow{2}{*}{$\begin{array}{c}\text { Costo por Kg } \\
\quad \$ \text { Cop }\end{array}$} & \multicolumn{2}{|r|}{$\mathrm{T} 1$} & \multicolumn{2}{|c|}{$\mathrm{T} 2$} & \multicolumn{2}{|c|}{$\mathrm{T} 3$} \\
\hline & & $\begin{array}{l}\% \text { en } \\
\text { Dieta }\end{array}$ & $\begin{array}{l}\text { Valor en } \\
\$(\text { Cop })\end{array}$ & $\begin{array}{l}\% \text { en } \\
\text { Dieta }\end{array}$ & $\begin{array}{l}\text { Valor en } \\
\$(\text { Cop })\end{array}$ & $\begin{array}{l}\% \text { en } \\
\text { Dieta }\end{array}$ & $\begin{array}{c}\text { Valor en } \\
\$(\text { Cop })\end{array}$ \\
\hline Torta de Soya & 1020 & 20 & 204 & 20 & 204 & 20 & 204 \\
\hline Torta de Palmiste & 525 & 7 & 36,75 & 7 & 36,75 & 7 & 36,75 \\
\hline Gluten de Maíz & 1320 & 1,5 & 19,8 & 1,5 & 19,8 & 1,5 & 19,8 \\
\hline Harina de Pescado & 2850 & 30 & 855 & 0 & 0 & 0 & 0 \\
\hline $\begin{array}{l}\text { Harina Subproductos } \\
\text { Avícolas }\end{array}$ & 2035 & 0 & 0 & 30 & 610,5 & 30 & 610,5 \\
\hline Harina de Trigo & 1005 & 7 & 70,35 & 7 & 70,35 & 7 & 70,35 \\
\hline Maíz Amarillo & 690 & 4 & 27,6 & 4 & 27,6 & 4 & 27,6 \\
\hline Harina de Yuca & 1200 & 2 & 24 & 2 & 24 & 2 & 24 \\
\hline Gelatina & 17400 & 0 & 0 & 2,9 & 504,6 & 2,1 & 365,4 \\
\hline Alfa celulosa & 14000 & 20,6 & 2884 & 20,6 & 2884 & 20,6 & 2884 \\
\hline Aceite Vegetal & 3445 & 3,7 & 127,465 & 0 & 0 & 0 & 0 \\
\hline Vitaminas $\mathbb{R}$ & 20880 & 2,1 & 438,48 & 2,5 & 522 & 2,5 & 522 \\
\hline 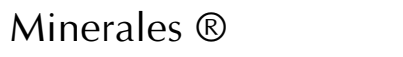 & 20880 & 2,1 & 438,48 & 2,5 & 522 & 2,5 & 522 \\
\hline Treonina & 5220 & 0 & 0 & 0 & 0 & 0,6 & 31,32 \\
\hline Lisina & 5046 & 0 & 0 & 0 & 0 & 0,1 & 5,046 \\
\hline Metionina & 9628 & 0 & 0 & 0 & 0 & 0,1 & 9,628 \\
\hline $\begin{array}{l}\text { Costo Kg de Dieta } \\
\$(\text { Cop) }\end{array}$ & & 512 & 5,925 & & 25,6 & & 32,394 \\
\hline
\end{tabular}




\section{Discusión}

La inclusión de fuentes alternativas de proteína, como harina de subproductos avícolas, harina de hueso, harina de sangre, sustitutos vegetales, entre otro, que reemplacen la harina de pescado ha sido investigada en numerosas especies acuícolas, donde los autores han coincidido en concluir que si es posible el reemplazo parcial o total de la harina de pescado sin general efectos negativos en los parámetros productivos (Schuchardt 2002, Yong 2004, Requeni 2005, Priya et al., 2007, Gutierrez-Espinosa 2010, Hernandez et al., 2010).

La tasa específica de crecimiento no fue afectada por el uso de esta fuente alternativa de proteína. Hernandez et al. (2010) reportaron resultados similares en alevinos de Oreochromis niloticus alimentados con dietas que contenían harina de subproductos avícolas. Priya et al. (2007) muestran una mayor tasa específica de crecimiento en Tilapia; diversos autores (Steffens 1994, Nengas et al. 1999, M.N. 2007, Fuertes et al., 2013) mostraron que no se afecta la tasa de crecimiento en Onchorhynchus mykiss y Sparus aurata. Menghong et al. (2008) indicaron que la suplementación con lisina y metionina puede aumentar el crecimiento en Carassius auratus gibelio. Contrario a lo anterior, en este investigación se evidenció que la suplementación de aminoácidos no resultó en mejor tasa de conversión; lo que a su vez muestra que a la conversión puede ser disminuida por la suplementación, concordando con $\mathrm{Yu}$ (2004).

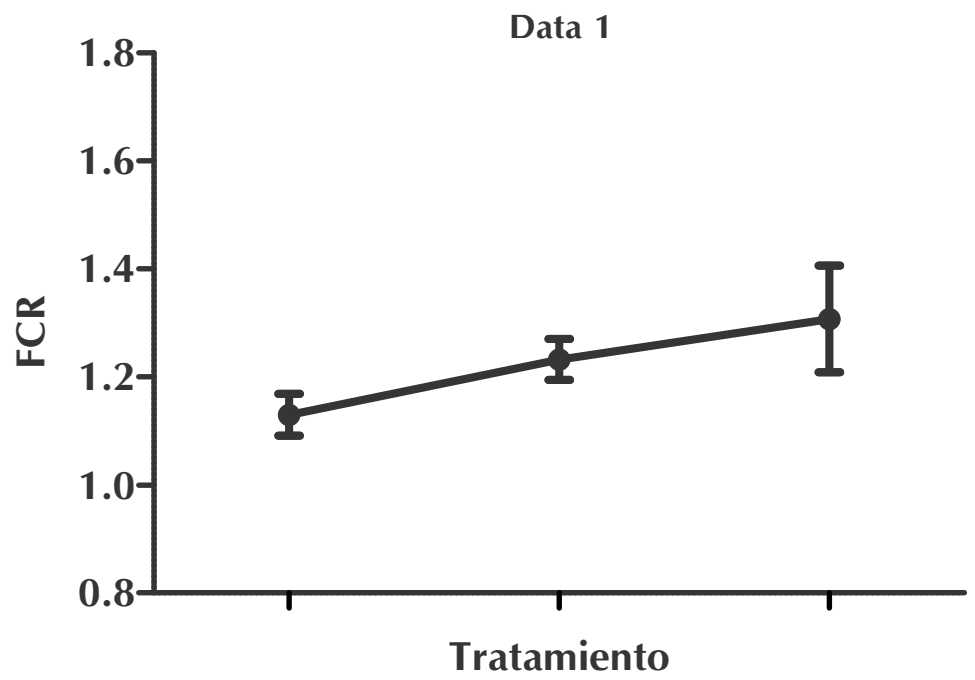

Figura 2. Tasas de Conversión alimenticia en los diferentes tratamientos

Los resultados de este experimento mostraron que la tasa de conversión alimenticia no se ve afectada al usar harina de subproductos avícolas (versus harina de pescado), y concuerdan con las investigaciones hechas en otras especies Yu (2004), Hernandez et al. (2010) (Figura 2). Priya et al., (2007) muestran que para Oreochromis niloticus esta tasa es mejor cuando son alimentadas con dietas que contienen harina de subproductos avícolas, que con dietas compuestas por harina de pescado $\mathrm{u}$ otros ingredientes proteicos de origen animal, como harina de sangre y harina de plumas. En cuanto a la ganancia de peso no se observó diferencias significativas entre los tratamientos, lo que supone quela suplementación con aminoácidos no tuvo efecto en este parámetro para cachama blanca. En trabajos realizados por Priya et al. (2007) y Hernandez et al. (2010) en 
Oreochromis niloticus, encontraron que los peces alimentados con la dieta que contenía harina de pescado obtuvieron ganancias de peso superiores a los alimentados con dietas que usan otras alternativas de proteína, incluida entre ellas la harina de subproductos avícolas. Webster et al. (1999) [en Menghong et al. 2008] indica que al suplementar con lisina, metionina y treonina, se puede incrementar la utilización de los nutrientes, aumentando la ganancia de peso en "sunshinebass" (Moronechrysopsx M. saxatilis). Subhadra et al. 2006 no encontraron diferencias para este parámetro en lubina negra, Micropterus salmoides. Rossi Jr et al. (2012) sugirieron hacer mezclas de ingredientes proteicos para Trachinotus carolinus para no disminuir la ganancia de peso. Al contrario de otros estudios (Abdel, et al., 2001, Turker, et al., 2005, Rossi Jr, et al., 2012), en el presente se observó que la harina de subproductos avícolas puede llegar a ser igual o más palatable que la harina de pescado, aumentando el consumo de alimento. Cabe anotar que la adición de aminoácidos provocó un aumento en el consumo de alimento; en el caso de la tilapia también se ve aumentado el consumo de las dietas con harina de subproductos avícolas (Priya, et al., 2007) y en alevines no presentan diferencias significativas (Hernandez, et al., 2010), concordando esto con investigaciones hechas en lubina negra (Subhadra, et al., 2006).

En los costos, se observa cómo sigue la tendencia de menor valor en la dieta elaborada con harina de pescado (T1). Resalta que la suplementación con aminoácidos en las dietas que contenían harina de subproductos avícolas no tiene que ser más costosa para su implementación, debido a que esta presenta una elevación del 4\% (T3) que resulta menor que en las dietas comparado con la dieta T2, que fue de $5.8 \%$. Este aumento puede deberse al uso de ingredientes semi-purificados, que en la vida práctica pueden ser cambiados por elementos de menor valor, que pueden dejar a las dietas a base de harina de subproductos avícolas como una alternativa más económica que la harina de pescado.

Se concluye que la harina de subproductos avícolas puede sustituir a la harina de pescado como ingrediente proteico en las dietas para Piaractus brachypomus. Los resultados no mostraron diferencia para ganancia de peso, tasa de conversión alimenticia y tasa de crecimiento especifico. Además, se evidencia que al no haber diferencias entre los tratamientos que contenían harina de subproductos avícolas suplementada y los que no, la suplementación con aminoácidos para estas dietas en la especie no sería recomendable, ya que acarrea más gastos sin un beneficio considerable en la producción. AI contrario, puede llevar a un aumento en el consumo de alimento, lo que repercutiría de forma negativa en los parámetros productivos. Los costos de las dietas dependen de las condiciones del mercado de cada uno de los ingredientes que las compongan, pero para el presente comparativo se demostró que el aumento en el precio puede llegar a ser sobrellevado con los parámetros productivos o por medio de convenios con las casas productoras de materias primas, para disminuir los costos de las mismas.

\section{Agradecimientos}

A la Dirección General de Investigaciones -DGI - de la Universidad de los Llanos por la cofinanciación del proyecto. 


\section{Referencias}

Abimorad EG, Carneiro DJ. Métodos de coleta de fezes edeterminação dos coeficientes de digestibilidade da fração protéicae da energia de alimentos para o pacu, Piaractus mesopotamicus (Holmberg, 1887). Rev Bras Zootec. 2004; 33:1101-1109. DOI: 10.1590/S1516-35982004000500001.

Abdel-Warith AA, Russell PM, Davies SJ."Inclusion of a commercial poultry byproduct meal as a protein replacement of Fish meal in practical diets for African cat fish Clarias gariepinus (Burchell 1822)." Aquac Res. 2001; 32(Suppl 1): 296-305.

Albert GJ Tacon, Marc Metian. Fishing for Feed or Fishing for Food: Increasing Global Competition for Small Pelagic Forage Fish. AMBIO A Journal of the Human Environment. 2009; 38(6): 294-302 DOI: 10.1579/08-A-574.1

Amaya EA, Rouse DB. "Replacement of Fish Meal in Practical Diets for the Pacific white shrimp (Litopenaeus vannamei) reared under pond Conditions." Aquaculture. 2007; 262: 393-401.

AOAC.1995. "Association of Official Analytical Chemists. Official Methods of Analysis 16th. AOAC 0066-961X, Arlington, Va".

De la Higuera M.1985. Fuentes de proteína y de energía alternativas en acuícultura Seminario sobre avances tecnológicos y necesidades en acuicultura, organizado por la ASA, Madrid, Asa.

Díaz F, López R. 1995. "El cultivo de la Cachama Blanca (Piaractus brachypomus) y de la cachama negra (Colossoma macropomum): Fundamentos de acuicultura continental." Instituto nacional de pesca y acuicultura (INPA): 207-221.
Fuertes JB, Celda JD, Carral JM, Saéz-Royuela M, González-Rodríguez A. "Replacement of fish meal with poultry by-product meal in practical diets for juvenile crayfish (Pacifastacus leniusculus Dana, Astacidae) from the onset of exogenous feeding " Aquaculture. 2013; 404-405: 22-27.

Gomez-Requeni P. 2005. Tesis "Fuentes Alternativas de Proteínas en Acuicultura. Disfunciones Endocrinas, Metabólicas e Inmuno-histopatológicas", UNIVERSITAT DE VALENCIA.

Gutiérrez-Espinosa MC, Vásquez-Torres W. Digestibilidad de Glicine max L, soya, en juveniles de Cachama Blanca Piaractus brachypomus, Cuvier 1818. Revista Orinoquía. 2008; 12(2):141-148.

Gutierrez-Espinosa M. 2010. Efecto de la sustitución de la harina de pescado por materias primas alternativas en la digestibilidad, crecimiento y retención de proteína y energía en tilapia nilótica, Oreochromis niloticus, Linnaeus 1758. Instituto de Acuicultura de los Llanos, Universidad de los Llanos. Maestria.

Hernandez C, Olvera-Novoa MA, Hardy RW, Hermosillo A, Reyes C,González B.2010. "Complete replacement of fish meal by porcine and poultry by-product meals in practical diets for fingerling Nile tilapia Oreochromis niloticus: digestibility and growth performance." Aquaculture Nutrition. 16(1): 44-53.doi : 10.1111/j.1365-2095.2008.00639.x

Alexis MN. 2007. "Fish meal and fish oil replacers in Mediterranean marine fish diets."CIHEAM 22: 183-204.

Alexis MN. Fish meal and fish oil replacers in Mediterranean marine fish diets. In : Tacon A.G.J. (ed.), Basurco B. (ed.). Feeding 
tomorrow's fish. Zaragoza : CIHEAM, 1997. p. 183-204.

Martínez CA, Chávez-Sánchez PM, OlveraNovoa MA, Abdo de la Parra MI.1996. Fuentes Alternativas de Proteínas vegetales como Sustitutos de La Harina de Pescado para la Alimentación en Acuicultura. Avances en Nutricion Acuicola III. Sinaloa, Mexico, UANL.p. 279-324.

Mendoza R, Aguilera C, Montemayor J. 2000. Utilización de subproductos avícolas en las dietas para organismosacuáticos. pp 398439 En: Civera-Cerecedo, R., PérezEstrada, C.J., Ricque-Marie, D. y CruzSuárez, L.E. (Eds.)Avances en Nutrición Acuícola IV. Memorias del IV Simposium Internacional de Nutrición Acuícola. Noviembre 15-18,1998. La Paz, B.C.S., México.

Menghong H, Youji W, Qian W, Min Z, Bangxi X, Xuegiao Q, et al."Replacement of fish meal by rendered animal protein ingredients with lysine and methionine supplementation to practical diets for gibel carp, Carassius auratus gibelio." Aquaculture. 2008; 275(1-4): 260-265.

Mesa-Granda MN, Botero-Aguirre MC."La Cachama Blanca (Piaractus brachypomus), una especie potencial para el mejoramiento genético." Rev Colom Cienc Pecua. 2007; 20(1):79-86.

Nengas I, Alexis MN, Davies SJ. "High inclusion levels of poultry meals and related by products in diets for gilthead seabream Sparus aurata L." Aquaculture. 1999; 179(1-4): 13-23.

Orozco JJ. 1990. "Estudio de crecimiento y de producción de cachama negra (Colossoma macropomum) y la cachama negra (Colossoma bidens) a densidades altas en tanques y jaulas flotantes." Informe CERER$\mathrm{U}: 42$.

Priya E, Davies S. "Growth and feed conversion ratio of juvenile Oreochromis niloticus fed with replacement of fishmeal diets by animal by-products." Indian J Fish. 2007; 54(1): 51-58.

Robinson E. 1990. "Use of cottonseed meal in catfih feeds." Aquaculture Magazine, March-April: 65-68.

Rossi Jr W, Davis DA."Replacement of fishmeal with poultry by-product meal in the diet of Florida pompano Trachinotus carolinus L." Aquaculture. 2012; 338-341: 160-166.

Schuchardt DRL, Fernandez-Palacios H, Hernández-Cruz CM, Valencia A, Vergara JM.2002. "SUSTITUCIÓN PARCIAL DE HARINA DE PESCADO POR HARINA DE KRILL EN DIETAS DE ENGRODE PARA BOCINEGRO (Pagrus pagrus)".

Steffens W. "Replacing Fish Meal with poultryby product meal in diets for Rainbow trout, Onchorhynchus mikiss " Aquaculture.1994; 124: 27-34.

Subhadra B, Lochmann R, Rawles S, Chen R."Effect of fish-meal replacement with poultry by-product meal on the growth, tissue composition and hematological parameters of largemouth bass (Micropterus salmoides) fed diets containing different lipids." Aquaculture. 2006;260(1-4): 221 231.

Turker A, Yigit M, Ergun S, Karaali B, Erteken A."Potential of Puoltry by-product meal as a substitute for fish meal in diets for Black Sea Turbot Scophthalmus maeoticus growth and nutrient utilization in winter." Isr J Aquacult Bamid. 2005; 57(1): 49-61. 
Webster CD, Tiu LG, Morgan AM. "Effect of partial and total replacement if fish meal on growth and body composition of sunshine bass Morone chrysops $\times$ M. Saxatilis fed practical diet." World Aquaculture. 1999;30(4): 443-453.

Yong Y, Xie S, Lei W, Zhu X, Yang Y. "Effect of replacement of fish meal by meat and bone meal and poultry by-product meal in diets on the growth and immune response of Macrobrachium nipponense." Fish Shellfish Immunol. 2004;17(2): 105-114.

Yu Y. 2004. Replacement of Fishmeal with Poultry by Product meal and meat and bone meal in shrimp, tilapia and trouts diets.Avances en Nutrición Acuícola VII. Memorias del VII Simposium Internacional de Nutrición Acuícola. Hermosillo, Sonora, México: 16-19. 